\title{
Lymphangiomes de langue de diagnostic difficile traités par vaporisation laser $\mathrm{CO} 2$.
}

\section{Bercault B1, Catros S1, Fricain JC1}

1. Médecine Bucco-Dentaire - CHU Bordeaux - Université Victor Segalen - Bordeaux II

\section{Introduction}

La malformation lymphatique (ML), anciennement appelée lymphangiome est une malformation bénigne des vaisseaux lymphatiques de nature hamartomateuse. II est présent presque exclusivement chez l'enfant de moins de deux ans, et est extrêmement rare chez l'adulte. Ces malformations peuvent siéger dans n'importe quelle région du corps, mais elles sont souvent retrouvées au niveau de la tête et du cou. Dans la cavité buccale, la langue est le site le plus fréquent. Ils sont considérés comme une séquestration du tissu lymphatique qui a conservé son potentiel de croissance. Trois variantes ont été décrites pour ces lymphangiomes (1) : le lymphangiome capillaire, caverneux et kystique. Certaines formes cliniques posent des problèmes diagnostiques.

\section{Observation}

Nous rapportons le cas d'un patient de 44 ans présentant des lymphangiomes superficiels microkystiques de la langue de diagnostic clinique et anatomopathologique difficile. II ne présentait aucun antécédent particulier. La symptomatologie avait débuté il y a plus de 1 an par l'apparition progressive de vésicules et papules blanchâtres de quelques millimètres, bien limitées, du bord antérieur et de la pointe de langue. Ces lésions évoluaient par poussées inflammatoires. Lors des crises, il y avait une majoration du volume des papules occasionnant des douleurs intenses (EVA 9/10), un œdème lingual ainsi que des saignements. Ces poussées inflammatoires survenaient toutes les 6 semaines avec une durée de 24 à 48 heures. Le bilan biologique était normal. Une première biopsie a été réalisée mettant en évidence un aspect papillomateux avec des zones inflammatoires congestives et de nécroses non spécifiques. Un traitement symptomatique par Dermoval crème a été prescrit. Devant la persistance de ces lésions à 6 mois, une nouvelle biopsie a été réalisée et le traitement par Dermoval a été arrêté. L'aspect histologique était en faveur d'une composante lymphangiomateuse sur un fond inflammatoire. Un traitement par laser CO2 a été initié avec succès. La surveillance régulière n'a révélé aucune récidive, avec un recul de 3 mois.

\section{Discussion}

Le lymphangiome kystique est une tumeur bénigne connue habituellement par sa survenue à un âge très précoce. 80 à $90 \%$ sont diagnostiquées vers l'âge de trois ans, leur survenue chez les adultes est très rarement constaté (2). ॥| est plus fréquemment rencontré au niveau cervical. La survenue au niveau de la langue n'est pas rare mais notre cas est atypique par sa localisation et l'aspect des lésions dont l'aspect kystique n'était pas évident. Les rares cas décrits au niveau de la langue, intéressent le dos de la langue, plus précisément en arrière du $V$ lingual. Le patient présentait uniquement des lésions du bord antérieur et de la pointe de langue. Ces lésions se présentaient sous forme de vésicules $<3 \mathrm{~mm}$.Le traitement des lymphangiomes kystiques de la cavité buccale repose sur la chirurgie qui consiste en une exérèse complète des kystes afin d'éviter les récidives. D'autres traitements sont parfois proposés : laser, sclérothérapie, ablation et cautérisation par radiofréquence, dans le but de déclencher une fibrose secondaire. II est important que le patient comprenne que cette pathologie est bénigne et qu'elle ne présente pas de risque de transformation maligne.

bercault787@gmail.com

(C) The authors, published by EDP Sciences. This is an Open Access article distributed under the terms of the Creative Commons Attribution License 4.0 (http://creativecommons.org/licenses/by/4.0/). 